\title{
Verteilungsmuster der subchondralen Mineralisierung in der Cavitas Glenoidalis bei Normalpersonen, Sportlern und Patienten
}

\author{
M. Müller-Gerbl ${ }^{l}, R \cdot$ Putz $^{l}, R \cdot K_{\text {Kenn }}^{2}$ \\ ${ }^{1}$ Anatomische Anstalt der Ludwig-Maximilians-Universität München \\ ${ }^{2}$ Radiologische Abteilung der Chirurgischen Klinik Innenstadt der Ludwig-Maximilians-Universität München
}

Herrn Prof. Dr. med. H. Frick aus Anlaß seines 70. Geburtstages gewidmet

\section{Zusammenfassung}

Um näheren Einblick in die Belastung des Schultergelenkes zu erhalten, wurde mittels der CTOsteoabsorptiometrie die Verteilung der subchondralen Mineralisierung in der Cavitas glenoidalis bei Normalpersonen, Sportlern und Patienten untersucht. Während sich bei jüngeren Normalpersonen 2 Dichtemaxima, ventral und dorsal, finden, zeigen alte Menschen ein zentrales Maximum, was auf eine unterschiedliche Mechanik infolge altersmäßiger Abschwächung der physiologischen Inkongruenz hindeutet. Bei Turnern ist die Gesamtmineralisierung signifikant erhöht, Maxima finden sich zentral oder nach dorsal verschoben. Bei Patienten mit habitueller Schulterluxation oder Instabilität sind die Dichtemaxima in die Randbereiche verlagert und deuten damit auf einen exzentrischen Einfall der Resultierenden hin. Mittels der CT-Osteoabsorptiometrie können diese individuellen Mineralisierungsmuster zur Darstellung gebracht und Informationen über die individuelle Beanspruchungssituation eines Gelenkes gewonnen werden.

\section{Distribution Pattern of Subchondral Mineralisation in the Glenoid Cavity in Ordinary People, Athletes and Patients}

To get information on the stresses acting on the shoulder joint, the distribution of subchondral mineralisation was examined by means of CT-Osteoabsorptiometry in the glenoid cavity in healthy people, athletes and patients. In young persons two density maxima are found ventrally and dorsally older persons, however, show a centrally located maximum which suggests a different joint mechanics in different ages depending on the decreasing physiological incongruence. In gymnasts the overall mineralisation is significantly higher, maxima are found centrally or shifted dorsally. In patients with recurrent dislocation of the shoulder or instability the zone of highest density is shifted to the edges of the glenoid cavity indicating that the prevailing position of the resultant force is excentrically. By means of CT-Osteoabsorptiometry these individual patterns of mineralisation can be displayed in the living and can give information on the individual mechanical situation ("loading history") of a joint.

\section{Einleitung}

Viele diagnostische Entscheidungen des klinisch-orthopädischen Alltages beruhen nicht zuletzt auf den Pauwels'schen Vorstellungen einer kausalen Beziehung zwischen hauptsächlicher lokaler Spannung im Gelenk und subchondraler Mineralisierung (Pauwels, 1965). Ein gut bekanntes Beispiel dafür sind die Formen der subchondralen Knochenschicht des Hüftpfannendaches, die im normalen AP-Röntgenbild bei Vorliegen von verschiedenen Krankheitsbildern wie beispielsweise Coxa valga oder vara ein typisches Erscheinungsbild aufweisen, das sich von dem einer gesunden Hüfte diagnostizierbar deutlich unterscheidet (Pauwels, 1973). Diese Veränderungen werden zwar als präarthrotische Zustände eingestuft, waren aber einer objektiven Bewertung bis dato nicht zu-

Z. Orthop. 131 (1993) 10-13

(C) 1993 F. Enke Verlag Stuttgart gänglich, da die konventionellen AP-Röntgenaufnahmen Summationsbilder darstellen, die keine Aussage über die Dichteverteilung in der Fläche erlauben.

Wir haben deshalb mit der CT-Osteoabsorptiometrie (CT-OAM) ein Verfahren entwickelt (Müller-Gerbl et al., 1989, 1990), mit dem es möglich ist, die flächenhafte Verteilung der subchondralen Knochendichte in Gelenkflächen auch beim Lebenden darzustellen, um damit erstens einen Einblick in die Abläufe der normalen Anpassung an die Gelenkmechanik und zweitens möglicherweise ein objektives Verfahren zur Beurteilung präarthrotischer Zustände zu erhalten.

\section{Ziel dieser Studie}

Darstellung subchondraler Mineralisierungsmuster in der Cavitas glenoidalis bei gesunden Normalpersonen, Leistungsturnern und Schulterpatienten. 


\section{Material}

CT-Datensätze mit einer Schichtdicke von $2 \mathrm{~mm}$ von 20 jungen (22-55 Jahre) und 20 älteren Personen (60-92 Jahre) ohne Beschwerden im Schulterbereich, von 11 Spitzenturnern (16-29 Jahre), die über mindestens 5 Jahre ein intensives Leistungstraining absolvierten, und von 12 Patienten (34-56 Jahre) mit diversen Schultererkrankungen (5 Fälle mit Instabilität des Schultergelenkes und 5 Fälle mit habitueller Schulterluxation). Bei zwei weiteren Patienten mit traumatischer Luxation lag der Humeruskopf aufgrund nicht erfolgter Reposition seit 1,5 bis 3 Monaten außerhalb der Pfanne.

\section{Methoden}

A. CT-Osteoabsorptiometrie (MüllerGerbl et al., 1989, 1990).

Die Auswertung nach der HounsfieldDichtestufenskala erfolgte mit dem in der Strahlentherapie eingesetzten Strahlentherapieplanungsrechner EVADOS. Nach Vergrößerung der interessierenden Gelenkflächen erfolgte die Konvertierung ins SIDOS-TELE-Format, das anschließend die zur Auffindung von Dichtebereichsgrenzen konzipierte Routine starten läßt. Durch Eingabe festzulegender Hounsfieldwerte erzeugt die SIDOS-TELE-Subroutine Isodensiten, d.h. in sich geschlossene Linien gleicher Dichte, die die Regionen größerer Dichte von denen kleinerer trennen. Diese Bilder werden anschließend mit bildanalytischen Methoden weiterverarbeitet und die Dichtebereiche in einer Falschfarbenzuordnung deutlich gemacht.

Für eine Projektion der subchondralen Dichte auf die Gelenkfläche wurden die Dichtewerte an den Einzelschnitten in einer definierten Tiefe von 1,5 mm gemessen und in eine Flächenkarte der Gelenkkontur übertragen, wodurch erst eine vergleichende Analyse der Spannungsverteilung über die gesamte Gelenkfläche möglich wird.

(Abb. 1).

\section{B. CT-OAM mittels 3-D-Rekonstruktion}

Eine Fortentwicklung der Methode wurde durch Einsatz eines CT-Programmes zur dreidimensionalen Rekonstruktion erreicht, wodurch die Dichteverteilung der subchondralen Mineralisierung schon am Computertomographen selbst dargestellt werden kann. Mit diesem Programm können sowohl die Gelenkfläche selbst wie auch die diversen Dichtestufen getrennt aufgebaut werden. Anschließend erfolgt mittels eines Bildanalysesystems die Darstellung der Dichtestufen in Falschfarben und deren Übereinanderprojektion.

Der Fortschritt bei dieser Vorgehensweise liegt darin, daß über das bisherige Verfahren hinaus die Projektion der subchondralen Dichte exakt auf die individuelle Form der Gelenkfläche unter einem erheblich verringerten Zeitaufwand bezogen werden kann.

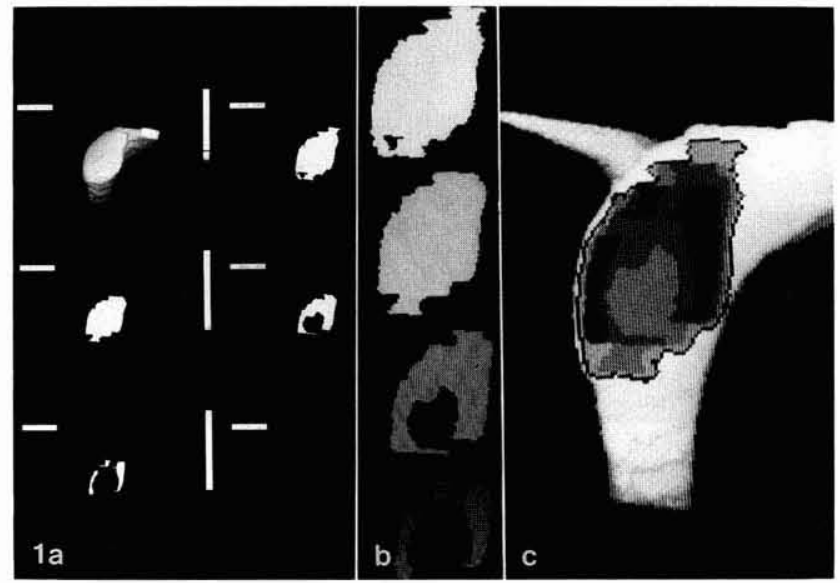

Abb. 1 Methodik der CT-Osteoabsorptiometrie (CT-OAM) mittels 3-D-Programm

a. Bilder der Cavitas glenoidalis und der einzelnen Dichtestufen, die am CT-Computer selbst entstehen

b. Falschfarbendarstellung der einzelnen Dichtestufen

c. Resultat nach Übereinanderprojektion

\section{Ergebnisse}

1. Normalpersonen: Bei jüngeren Pesonen finden sich 2 Dichtemaxima jeweils im ventralen und dorsalen Bereich der Cavitas glenoidalis. Die Dichtekarten älterer Personen dagegen zeigen meistens ein Dichtemaximum im zentralen Anteil der Cavitas glenoidalis (Abb. 2).

2. Turner: Bei allen Turnern ist die Dichte insgesamt deutlich erhöht und breitflächig zentral lokalisiert (Abb. 3). Bei etwa einem Drittel findet sich eine Verschiebung des Dichtemaximums an den dorsalen Rand.

3. Patienten: Patienten mit Schulterinstabilität oder habitueller Schulterluxation weisen in 8 von 10 untersuchten Fällen ein geändertes Dichtemuster auf. Die Dichtemaxima sind in die Randbereiche verlagert (Abb. 3).

Die 2 Patienten mit längerzurückliegender traumatischer Luxation ohne Reposition zeigen eine signifikant erniedrigte Gesamtmineralisierung (Abb. 3).

\section{Diskussion \\ I. Mechanik des Schultergelenkes und Arthrose-Entstehung}

Der Vergleich der Ergebnisse bei jungen und älteren Normalpersonen weist auf eine altersabhängig unterschiedliche mechanische Situation im Schultergelenk hin und ergänzt die Befunde unserer früheren Studie an einer Präparateauswahl von Menschen höheren Lebensalters (Müller-Gerbl et al., 1990), bei denen zentrale Mineralisierungsmaxima gefunden wurden. Die Altersabhängigkeit der Mineralisierung entspricht Befunden im Acetabulum des Hüftgelenkes. Dort sind, wie Bullough (1968, 1973) u.a. zeigte, beim jüngeren Menschen die Gelenkkörper inkongruent, diese Inkongruenz nimmt aber mit zunehmenden Alter ab. Studien über die Kontaktflächen ergaben beim jüngeren Menschen dementsprechend Kontaktzonen im vorderen und hinteren Pfannenbereich, bei 


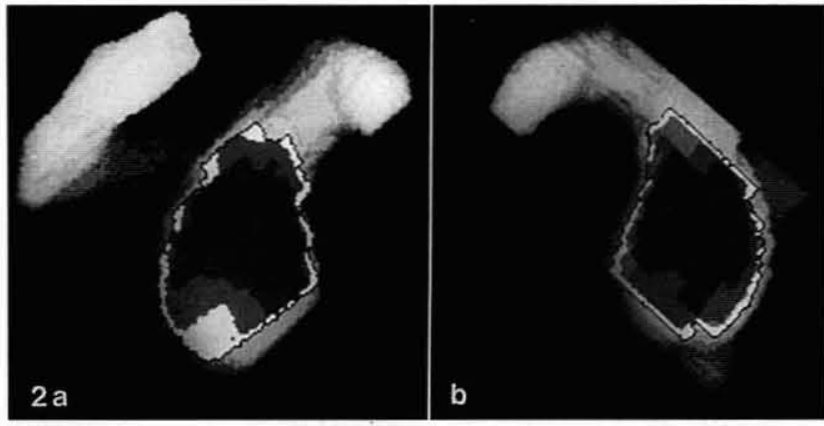

Abb. 2 Dichtekarten der Cavitas glenoidalis bei Normalpersonen (Ansicht von lateral)

a. 24jähriger Mann

b. 65jährige Frau

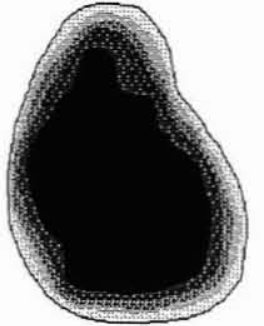

a

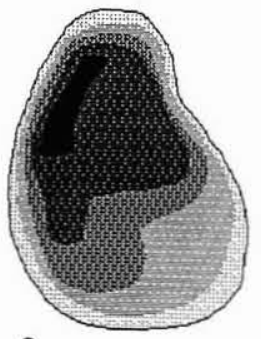

C

Abb. 3 Dichtekarten der Cavitas glenoidalis (Ansicht von lateral) bei

a. Turner

b. Patient mit 3 Monate zurückliegender traumatischer Luxation ohne Reposition des Humeruskopfes

c. 36jähriger Patient mit habitueller Schulterluxation

d. 47jährige Patientin mit vordere Schulterinstabilität

älteren Personen dagegen zentral. U.a. konnte Miyanaga (1984) darüberhinaus eine Abhängigkeit der Ausdehnung und der Lokalisation der Kontaktflächen, d.h. dem Grad der Inkongruenz, von der tatsächlich wirkenden Gelenkskraft nachweisen. Demnach kommt es auch bei primär inkongruenten Gelenkflächen ab einer bestimmten Größe der einwirkenden Gelenkkraft zu einem vollständigen Kontaktschluß mit nachfolgend größerer Kontaktfläche und kleinerer Druckspannung, was Bullough (1981) in einem Modell verdeutlicht (Abb. 4). Die bei geringeren Belastungen vorliegende Inkongruenz ist allerdings positiv anzusehen, gewährleistet sie doch eine optimale Ernährung und Schmierung des Gelenkknorpels.

Ein abschätzender Vergleich der Größe der bei verschiedenen Tätigkeiten auftretenden Gelenkkräfte und der Häufigkeit, mit der diese Tätigkeiten während ei-

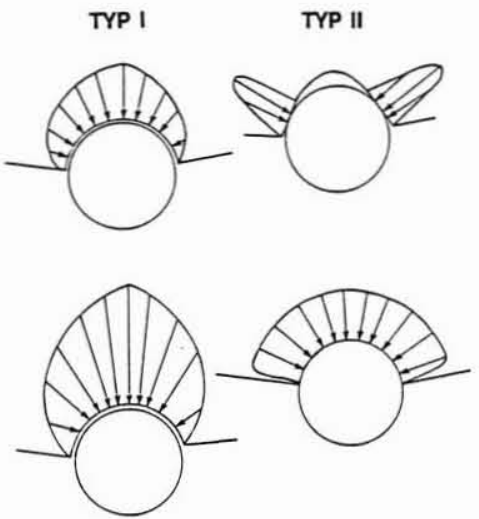

Abb. 4 Modell der Spannungsverteilung eines $\mathrm{Ku}$ gelgelenkes (aus 1981) Gelenk (Situation beim älteren Menschen)

b. Inkongruentes Gelenk (Situation beim jüngeren Menschen) Bullough et al.,

a. Kongruentes a

b nes Tages ausgeübt werden, läßt den Schluß zu, daß nur etwa während $5 \%$ bis maximal $25 \%$ des Tages so große Gelenkkräfte auftreten, daß ein kompletter Kontaktschluß entsteht (Marsden, 1971). Konsequenzen sind zum einen eine erhöhte lokale Belastung in den vorderen und in den hinteren Anteilen, zum anderen aber auf Dauer eine relative Unterforderung des zentralen Pfannenbereiches. Der Knorpel dieses Gebietes weist tatsächlich häufig schon früh entsprechende degenerative Zeichen auf, die aber die Gelenkfunktion so lange nicht stören, als die Inkongruenz erhalten bleibt.

Es erscheint damit plausibel, daß die Bestandteile des normalen Schultergelenkes durch eine physiologische Inkongruenz so exakt konfiguriert sind, daß sie sowohl ideale Bedingungen für eine kraftabhängige, also dynamische Art der Kraftübertragung und -verteilung als auch für die Schmierung und Ernährung des belasteten Knorpels liefern. Bei alten Menschen geht diese Inkongruenz, die dem belasteten Knorpel eine akzeptable Umgebung gewährleistet, verloren und setzt einen nicht aufzuhaltenden Prozeß des Knorpelunterganges durch mangelnde Ernährung und Abrieb in Gang. In Folge wird der zentrale Pfannenbereich, der während des Zeitraumes der Inkongruenz wegen relativer Unterforderung degeneriert ist, zu einer ständigen Kontaktzone mit ensprechenden katastrophalen Konsequenzen für den humeralen und glenoidalen Gelenkknorpel mit nachfolgender Arthrose, was sich auch in der Häufigkeit der gefundenen Degenerationen im zentralen Pfannenbereich niederschlägt (Kohn, 1985).

$\mathrm{Da}$ auch für andere Gelenke ähnliche Befunde vorliegen, handelt es sich bei der Inkongruenz der Kugelgelenke offenbar um ein optimiertes biologisches Bauprinzip.

$\mathrm{Ob}$ nun die Inkongruenz auf primäre strukturelle Gegebenheiten des Knochens oder auf eine von einigen Autoren beschriebene funktionelle Knorpelschwellung (Ingelmark, 1948, Ekholm, 1952, Walker, 1973, Oberländer, 1978) zurückzuführen ist, kann noch nicht beantwortet werden. Genausowenig die Frage, ob die mit dem Alter abnehmende Inkongruenz durch knöcherne Veränderungen der Gelenkkörper oder durch eine nachlassende funktionelle Leistungsfähigkeit des Gelenkknorpels verursacht wird. 


\section{II. Über- bzw. Unterforderung des Schultergelenkes}

Bei den Turnern dagegen ist bekannt, daß in bestimmten Übungsphasen Kräfte bis zum Fünffachen des Körpergewichtes auftreten können (Bodem et al., 1984). Das bedeutet, daß hier infolge der häufig wirkenden großen Kräfte der zentrale Pfannenbereich überwiegend als Kontaktzone beansprucht wird und damit ein biologischer Anreiz zur Erhöhung der Mineralisierung in diesem Bereich entsteht.

Eine verminderte Mineralisierung als Folge einer Minderbeanspruchung zeigen die 2 Patienten, bei denen seit 3 Monaten eine nicht reponierte Schulterluxation vorliegt.

\section{Pathomechanik des Schultergelenkes}

Bei den meisten Patienten mit Instabilität oder habitueller Luxation treten vom Normalfall abweichende Dichtemuster mit einer Verlagerung der Maxima in die Randbereiche auf. Während die Befunde bei Normalpersonen auf einen zentralen Durchstoßpunkt der Gelenksresultierenden mit Auftreten von zwei zentralen Dichtemaxima aufgrund der bestehenden Gelenkinkongruenz hinweisen, läßt die Verschiebung der Dichtebereiche in die Randzonen eine auf Dauer exzentrische Lage der Resultierenden vermuten. Dadurch werden in den Randbereichen Spitzenspannungswerte erreicht, die auf Dauer zu einer Schädigung des Knorpels und des Knochens führen können und damit der Entstehung einer Arthrose im Sinne einer Überbeanspruchung Vorschub leisten können. Auch Schmerzsyndrome ohne sonstige morphologische Korrelate könnten darin ihre Erklärung finden.

\section{Zusammenfassung}

Es kann somit festgestellt werden, daß die innerhalb der Gelenkflächen der großen Gelenke bestehenden regelhaften reproduzierbaren Verteilungsmuster der subchondralen Mineralisierung durch entsprechende Umbauvorgänge des Knochens Ausdruck der jeweiligen mechanischen Situation im Sinne eines Spiegelbildes der sogenannten „loading history“, sind.

\section{Klinische Relevanz der CT-OAM}

Der Wert der CT-Osteoabsorptiometrie besteht darin, daß damit das morphologische Korrelat der hauptsächlichen Beanspruchung der Gelenke individuell am Lebenden dargestellt werden kann. Die klinische Relevanz dieser Methode beinhaltet ein weites Spektrum an Möglichkeiten. Sie kann zu diagnostischen Zwecken eingesetzt werden, um Aufschluß über die individuelle mechanische Situation eines Gelenkes zu erhalten. Sie bietet sich zum Einsatz in der klinischen Grundlagenforschung am Bewegungsapparat an, da sie eine wenig invasive, den Patienten nicht über Gebühr belastende Untersuchungsmethode darstellt. Damit steht u.E. eine Methode zur Verfügung, die in besonders gelenkbeanspruchenden Be- reichen der Arbeits- und Sportwelt für die Prävention und Früherkennung von Gelenkschäden und für eine objektive Bewertung von sogenannten präarthrotischen Zuständen eingesetzt werden kann, was angesichts der flächendekkenden Zahl von CT-Geräten nicht auf den Einzelfall beschränkt bleiben muß.

Um noch einen kleinen spekulativen Ausblick zu wagen, könnte sich aus dieser Methode speziell für den verantwortungsbewußten Kliniker eine ganz bestimmte Anwendung ergeben, nämlich die kritische Verlaufskontrolle aller gelenkbetreffenden Operationen, die eine Änderung der Gelenkmechanik unterstellen, wie beispielsweise die Umstellungsosteotomien bei Coxa vara und Coxa valga oder Operationen zur Entlastung des Femoropatellargelenkes.

\section{Literatur}

${ }^{1}$ Bodem, F., F. Brussatis, W. Menke: Zur theoretischen Biomechanik des Schultergelenkes: Die Entstehung gewöhnlicher und außergewöhnlicher mechanischer Belastungen des glenohumeralen Gelenkknorpels. In: Biomechanik der gesunden und kranken Schulter, Thieme, Stuttgart, 1984

2 Bullough, P., J. Goodfellow, A. S. Greenwald, J. O'Connor: Incongruent surfaces in the human hip joint. Nature 217 (1968) 1290

${ }^{3}$ Bullough, P., J. Goodfellow, J. O'Connor: The relationship between degenerative changes and load-bearing in the human hip. J. Bone Jt. Surg. 55B (1973) 746-758

${ }^{4}$ Bullough, P.: The geometry of diarthrodial joints, its physiologic maintenance, and the possible significance ofe age-related changes in the geometry-to-load distribution and the development of osteoarthritis. Clinical Orthopaedics and Related Research 156 (1981) 61-66

5 Ekholm, R., B. E. Ingelmark: Functional thickness variations of human articular cartilage. Acta Soc. Med. Ups. 57 (1952) 39-59

6 Ingelmark, B. E., R. Ekholm: A study on variations in the thickness of articular cartilage in association with rest periodical loading. An experimental investigation on rabbits. Acta Soc. Med. Ups. 53 (1948) 6174

${ }^{7}$ Kohn, D., W. Glaubitz, H. Schmidt, P. Lobenhofer: Lokalisation, Art und endoskopische Beurteilung degenerativer Veränderungen im Schultergelenk. In Biomechanik der gesunden und kranken Schulter. Hrsg. H. J. Refior, W. Plitz, M. Jäger, M. H. Hackenbroch, Thieme, Stuttgart (1985) 152-156

${ }^{8}$ Marsden, J. P., S. R. Montgomery: Plantar power for arm prothesis using body weight transfer. In Conference on human locomotor engineering, p. 260 . held on September $7-10,1971$, at the University of Sussex. London: Institution of Mechanical Engineers, 1971

${ }^{9}$ Miyanaga, Y., T. Fukubayashi, H. Kurosawa: Contact studies of the hip joint. Arch. Orthop. Trauma. Surg. 103 (1984) 13-17

${ }^{10}$ Müller-Gerbl, M., R. Putz, N. Hodapp, E. Schulte, B. Wimmer: Computed tomography-osteoabsorptiometry for assessing the density distribution of subchondral bone as a measure of long-term mechanical adaptation in individual joints. Skeletal Radiol. 18 (1989) $507-$ 512

${ }^{11}$ Müller-Gerbl, M., R. Putz, N. Hodapp, E. Schulte, B. Wimmer: Die Darstellung der subchondralen Dichtemuster mittels der CT-Osteoabsorptiometrie (CT-OAM) zur Beurteilung der individuellen Gelenkbeanspruchung am Lebenden. Z. Orthop. 128 (1990) 128-133

12 Oberländer, $W$.: On Biomechanics of joints, the influence of functional swelling on the congruity of regularly curved joints. J. Biomechanics 11 (1978) 151-153

${ }^{13}$ Pauwels, F.: Gesammelte Abhandlungen zur Biomechanik des Bewegungsapparates. Springer, Berlin-Heidelberg-New York, 1965

14 Pauwels, F.: Atlas zur Biomechanik der gesunden und kranken Hüfte. Springer, Berlin-Heidelberg-New York, 1973

15 Walker, P. S.: A comparison of normal and artificial human joints. Acta orthop. belg. 39 (1973) Suppl., 43-54

\section{Dr. M. Müller-Gerbl}

Anatomische Anstalt der LMU München

Pettenkoferstr. 11

8000 München 2 